\title{
Mindfulness-Based Stress Reduction (MBSR) Group Intervention to Reduce Burnout among Caregivers in Nursing Home
}

\author{
Risky Adinda \\ Faculty of Psychology, Department of Clinical Psychology, Universitas Indonesia \\ risky.adinda7I@ui.ac.id \\ Dini Rahma Bintari* \\ Faculty of Psychology, Department of Clinical Psychology, Universitas Indonesia \\ dini.rahma@ui.ac.id
}

\begin{abstract}
Professional caregivers are prone to experience burnout. Burnout is a condition of physical, emotional, and mental fatigue caused by prolonged stress due to work negatively impacts work performance and well-being of professional caregivers. The present study aims to explore the effectiveness of group intervention using Mindfulness-Based Stress Reduction in reducing burnout and enhancing psychological well-being of professional caregivers in a nursing home. Five caregivers (four males and one female) participated in four intervention sessions and a follow-up session. Maslach Burnout Inventory and Ryff's Psychological WellBeing Scale was used to measure burnout and psychological well-being, respectively. Qualitative data were also obtained through interviews at the end of the intervention. Statistical analysis using Wilcoxon Signed-Rank Test indicated that MBSR was not significantly effective in reducing burnout and enhancing psychological well-being. However, a trend of positive improvement was observed, indicating the potential benefits of MBSR for reducing burnout in professional caregivers. Limitations of this study are explained in the discussion section.
\end{abstract}

Keywords: Burnout, caregiver, mindfulness, psychological well-being

Received 18 February 2020/Accepted I3 May 2020 @JEHCP All rights reserved

\section{Introduction}

Burnout is a condition caused by prolonged stress due to work (Maslach, Schaufeli, \& Leiter, 200I), which consists of three main components: emotional exhaustion, depersonalization, and feeling of low personal accomplishment (Maslach \& Leiter, 2016). Burnout is often found in occupations related to human services or health, such as nurses and caregivers (Maslach et al., 200I). Caregivers provide care for clients who are usually in 
a vulnerable or critical condition, thus making the interaction between the two emotionally strenuous for the caregiver (Hasenfeld, 2010, in Lizano, 2015).

Caregiving profession found with high risk of experiencing burnout is professional caregivers for the elderly (Gosseries et al., 20I2). This is caused by the emotional burden in caring for clients with the characteristics of declining physical and cognitive functions and serious illness, as well as facing the client's death (Cocco, 20I0). Functional deteriorations and illness make elderly clients tend to be more dependent, thus require more attention and care from the caregiver (Cocco, 20l0). For professional caregivers, working in an institution specialized in elder care (e.g., nursing home) could increase the risk of experiencing burnout.

In Jakarta, as of December 2018 there were 5,309 elderly people accommodated as fostered residents in nursing homes governed by the Social Services (Jakarta Open Data, 2018). One of the nursing homes, Budi Mulia 3, accommodated 285 elderly as of July 2019. In contrast, there are 55 professional caregivers in Budi Mulia 3, making the ratio between the caregivers to elderly disproportionate. Based on previously conducted observation and informal interviews to caregivers in Budi Mulia 3, one professional caregiver can be responsible for one room with at least 10 elderly people. Ideally, a professional caregiver in his service should only serve up to 5 elderly people, to provide the best care (Depsos $\mathrm{RI}$ in Marsaoly, 200I). Budi Mulia 3 is also a social institution that accommodates elderly people from lower socioeconomic classes, most of which are neglected elderly without family or residence to go home to. The number of elderly people accommodated in Budi Mulia 3 increases every year, therefore increasing the workload of the caregivers who work there. Based on the interview, some caregivers reported feeling exhausted at the end of the day, lacking motivation and enthusiasm to work, complained about being unable to spend more time with their families on their day off because of the exhaustion, and sometimes had to work on their day off if there are any emergency in the institution. Their exhaustion and negative feelings are not only caused by their caregiving task, but also other tasks they have as employees in a social institution. Some caregivers also 
reported feeling angry and frustrated towards the elderly they care for. The high workload they have may ultimately increase their risk to higher work-related stress, and may lead to burnout.

Emotional exhaustion experienced in burnout refers to feeling overwhelmed and drained of physical and emotional energy (Maslach \& Leiter, 2016). As a result, feelings of depersonalization (negative feelings and attitudes towards their clients, inability to form a warm relationship with the clients) develop. Condition of burnout also decreases individuals' self-confidence related to their competence, and evaluate themselves negatively, thus developing a feeling of failure at their job (Maslach \& Leiter, 2016). There are various negative impacts from burnout. In addition to lowering the effectiveness of the individuals in working and providing services, it also has a negative impact on their psychological well-being, such as increasing anxiety, depression, lowering self-esteem, feelings of loneliness, and affecting interpersonal relationships (Shapiro, Brown, \& Biegel, 2007). Considering these negative impacts, it is important for professional caregivers to develop more effective stress management strategies to lower their stress at work and prevent burnout.

Many interventions to help alleviate burnout symptoms usually focus on stressmanagement or coping strategies, to prevent burnout, or prevent adverse consequences in employees already suffering from burnout (Ahola, Toppinen-Tanner, \& Seppänen, 2017). The intervention in this study used the Mindfulness-Based Stress Reduction approach (Kabat-Zinn, 2003). Mindfulness is most commonly defined as the state of full awareness and attentiveness to what is happening in the present, including thoughts, feelings, and physical sensations, with an open and accepting attitude (Brown \& Ryan, 2003). The exercise of mindfulness in MBSR encourages participants to be more attentive and develop a non-judgmental view of their thoughts, feelings, and physical sensations (Shapiro, Astin, Bishop, \& Cordova, 2005). MBSR was chosen due to its well-established model and one of the interventions proven to help reduce burnout in caregivers (Shapiro et al., 2005). MBSR is also cost-effective and time-efficient as it can be conducted as a brief 
program that can be implemented easily in hospitals or other healthcare providers (Shapiro et al., 2005; Nowrouzi et al., 2015). MBSR focuses on the practice of mindfulness. This helps participants to understand their own usual reactions to stress and promote healthier and more adaptive reactions (Shapiro et al., 2005).

Preceding studies on MBSR intervention for healthcare providers showed significant results of MBSR in decreasing stress and improving quality of life, self-compassion, and psychological well-being (Shapiro et al., 2005; Nyklíček \& Kuijpers, 2008). Practicing mindfulness was associated with MBSR's positive benefit of improving an individual's psychological well-being, as well as reducing various psychopathology symptoms, such as stress or mood disorders, along with improving overall mental health (Brown, Ryan, \& Creswell, 2007). Another study also found MBSR as an effective intervention to reduce burnout among nurses (Cohen-Katz, Wiley, Capuano, Baker, \& Shapiro, 2005; Mackenzie, Poulin, \& Seidman-Carlson, 2006).

Professional caregivers, especially who work at nursing homes and provide care for the elderly are prone to experience burnout, which negatively affects their psychological wellbeing. Therefore, it is important to provide interventions that can help professional caregivers regulate the stressto prevent burnout from prolonged stress that arises from their work. The present study is an intervention study that aims to examine the effectiveness of group intervention using MBSR to reduce burnout and improve psychological well-being among professional caregivers especially in nursing home institutions. Considering the negative effects of burnout, psychological well-being is also measured as an indicator of the effectiveness of the intervention. MBSR will be conducted as a group intervention, consisting of a therapist and several participants. 


\section{Method}

\section{Participants}

Participants were professional caregivers in Budi Mulia 3 Nursing Home, South Jakarta. This institution was chosen based on previous observation and interviews conducted by the researcher, that there was a gap between the numbers of the elderly $(N=285$, as of July 2019) and the professional caregivers $(N=55)$. Diverse characteristics of the elderly that require extra care; coupled with other tasks they have as employees, increase their workload and risk of experiencing burnout.

Participants in this study were five people who met the following criteria: (a) is a professional caregiver working in Budi Mulia 3 Nursing Home; (b) have low, medium, or high burnout score, measured using Maslach Burnout Inventory (Maslach, Jackson, \& Leiter, 1997); and (c) willing to fully participate in the group intervention consisting of four sessions. In the participant recruitment process, Budi Mulia 3 recommended ten caregivers to participate in the intervention, based on evaluation of their work performance. Researcher then contacted the recommended individuals, and five of them stated their willingness to join and fully participate all four sessions of the intervention. As for the other five recommended, one was unable to be contacted, and four others were unable to fully participate. The following table contains participants' information:

Table I

Participants Information

\begin{tabular}{lllll}
\hline Participant & Gender & Age & Duration of Work* & Status \\
\hline A & Male & 36 & 9 years & Married \\
B & Male & 28 & 7 years & Single \\
C & Male & 24 & 7 months & Married \\
D & Female & 20 & 5 months & Single \\
E & Male & 23 & 7 months & Single \\
\hline
\end{tabular}

All participants were each given a written informed consent, which guarantees privacy and confidentiality for all data obtained throughout the intervention process. The informed 
consent also ensures that participants may retract their agreement to participate, and retreat from the intervention should they feel uncomfortable, with notice to the therapist.

\section{Design}

This study used a quasi-experimental method with one group pre-post test design, where there was only one group of participants that received group intervention using Mindfulness-Based Stress Reduction. This design was used based on the agreements with Budi Mulia 3, where they asked for all of the agreeing participants to be given the same treatment. Pre-test and post-test measurements were carried out before and after the intervention program. Unstructured interviews were also given to all participants at the end of the intervention, to gain qualitative data and feedback on the intervention.

\section{Procedure}

The group intervention was conducted from 2 July 2019 to 25 July 2019 at the multipurpose hall in Budi Mulia 3 Nursing Home, South Jakarta. The intervention consisted of four sessions (twice a week, 2.5 hours each), which included the pre-test and post-test measurements. The intervention module was adapted from the MBSR manual created by Putri (2017) in a graduate thesis. A typical MBSR program consists of eight sessions (once a week, 60-180 minutes) (Putri, 2017). In the research, the manual had been adjusted by shortening the program into six sessions (including a pre-session and a follow-up session), and reducing some MBSR techniques that are more time-consuming. Thispresent intervention adapted the techniques used in the manual for time efficiency, to optimize the limited time allocated by Budi Mulia 3. Since the caregivers in Budi Mulia 3 cannot leave their work during the intervention process, we were given two weeks to carry out the intervention, which allows the participants to get back to work after every session ended.

Session I consisted of administering the informed consent forms, pre-test completion, group forming and commitment, group sharing about problems related to working as professional caregivers, "mindful check-in" meditation, and homework assignment. Session 
2 consisted of psychoeducation about stress, habitual style of thinking, and the MBSR principle, homework review, "mindful breathing" and "mindful self-inquiry" meditations, psychoeducation about and exploration on inner rule, and homework assignment. Session 3 consisted of homework review, "loving-kindness", "reflecting on others" and "is your body happy?" meditations, and homework assignments. Session 4 consisted of homework and session I through 3 reviews, practicing the mindfulness techniques from previous sessions, establishing post-intervention commitments and plans, post-test completion, and termination. Two weeks after the intervention ended, a follow-up session was conducted to check the conditions of participants, by completing the follow-up test and short interviews.

\section{Research Instruments}

Burnout was measured using Maslach Burnout Inventory, or MBI (Maslach et al., 1997). $\mathrm{MBI}$ was constructed to measure three main components of burnout: emotional exhaustion, depersonalization, and feeling of low personal accomplishment. MBI consists of 22 statements related to personal feelings or attitudes toward work or clients. Items in $\mathrm{MBI}$ are responded using a 7-point Likert scale $(0=$ never; $1=$ several times a year; 2 = once a month; 3 = several times a month; 4 = once a week; 5 = several times a week; and $6=$ every day). Scores obtained are interpreted differently for each of the burnout components, with details as follow: 
Table 2

Interpretation of Maslach Burnout Inventory

\begin{tabular}{lll}
\hline Component & Score & Interpretation \\
\hline Emotional Exhaustion & $\leq 17$ & Low \\
& $18-29$ & Medium \\
Depersonalization & $\geq 30$ & High \\
& $\leq 5$ & Low \\
Personal Accomplishment & $6-11$ & Medium \\
& $\geq 12$ & High \\
& $\geq 40$ & Low \\
& $34-39$ & Medium \\
& $\leq 33$ & High \\
\hline
\end{tabular}

It can be concluded that burnout condition is reflected by high scores of emotional exhaustion and depersonalization, and low score of personal accomplishment. All three components are scored and interpreted separately, making no total score of burnout.

Table 3

$\mathrm{MBI}$ Item Sample

\begin{tabular}{|c|c|c|}
\hline Domain & Item no. & Item sample \\
\hline Emotional exhaustion & I & I feel emotionally drained from my work \\
\hline Depersonalization & 5 & $\begin{array}{l}\text { I feel I treat some clients as if they were impersonal } \\
\text { objects }\end{array}$ \\
\hline Personal accomplishment & 19 & I have accomplished many worthwhile things in this job \\
\hline
\end{tabular}

In addition to burnout, psychological well-being was also measured as a secondary outcome. Psychological well-being was measured using Psychological Well-being Scale, Short Version (18 items) from Carol Ryff (Ryff \& Keyes, 1995). This version is shorter than its original 42 item-version. Six aspects of well-being and happiness is measured in PWBS, which includes autonomy, environmental mastery, personal growth, positive relationships, sense of purpose, and self-acceptance. Each aspect is represented by 3 items in PWBS. Items in PWBS are statements about self, and rated using a 6-point Likert scale, 
where lowest score is 18 and highest score is 108. Higher scores reflect higher level of psychological well-being. A sample statement in PWBS items is "For me, life has been a continuous process of learning, changing, and growth."

Quantitative assessment was obtained by comparing the levels of burnout and psychological well-being of each participant, collected from the research instruments completed in pre-test, post-test, and follow-up. Qualitative assessment was obtained through one-on-one interviews after the intervention ended. The interview was unstructured but focused on the following questions:

I. What the participants gained from the intervention

2. How the participants feel about themselves after the intervention

3. How the participants feel about their work as a caregiver and their clients

\section{Result}

In general, results (Table 3) showed that the average level of burnout across participants decreased, from the scores of $\mathrm{MBI}$ assessed before the intervention (pre-test) and after the intervention (post-test). Both emotional exhaustion and depersonalization showed a decrease in the average score, and personal accomplishment showed an increase in average score. The Wilcoxon Signed-Rank Test was carried out to evaluate whether these differences were statistically significant.

For each domain, the average emotional exhaustion level before intervention $(M=17.4)$ decreased after intervention $(M=13.6)$ by 3.8 points. This decrease, however, was not statistically significant $(Z=-.92, p=.35)$. The average depersonalization level before intervention $(M=5.8)$ also decreased after intervention $(M=3.6)$ by 2.2 points. The average personal accomplishment level before intervention $(M=37.8)$ showed an increase after intervention $(M=40.4)$ by 2.6 points. Analysis for the two domain showed the average depersonalization score in post-test is approaching significant lower than the pre- 
test $(Z=-1.82, p=.068)$, and the average personal accomplishment score in post-test is approaching significant higher than the pre-test $(Z=-1.82, p=.068)$.

Table 4

Comparison of Pre-test and Post-test MBI Scores

\begin{tabular}{|c|c|c|c|c|c|c|}
\hline \multirow{2}{*}{ Participant } & \multicolumn{2}{|c|}{ Emotional Exhaustion } & \multicolumn{2}{|c|}{ Depersonalization } & \multicolumn{2}{|c|}{ Personal Accomplishment } \\
\hline & Pre-test & Post-test & Pre-test & Post-test & Pre-test & Post-test \\
\hline$A$ & 4 & 2 & 1 & 0 & 48 & 48 \\
\hline B & 29 & 31 & 14 & 14 & 33 & 39 \\
\hline C & 20 & 19 & 4 & 1 & 31 & 32 \\
\hline $\mathrm{D}$ & 28 & 10 & 4 & 2 & 36 & 38 \\
\hline$E$ & 6 & 6 & 6 & I & 41 & 45 \\
\hline Mean & 17.4 & 13.6 & 5.8 & 3.6 & 37.8 & 40.4 \\
\hline Z & \multicolumn{2}{|c|}{-.92} & \multicolumn{2}{|c|}{-1.82} & \multicolumn{2}{|c|}{-1.82} \\
\hline Sig $(p<.05)$ & \multicolumn{2}{|c|}{.357} & \multicolumn{2}{|c|}{.068} & \multicolumn{2}{|c|}{.068} \\
\hline
\end{tabular}

In addition to comparing the average burnout level, results (Table 4) also showed all participants experienced an increase of psychological well-being score in average, from the PWBS assessed before intervention $(M=84.2)$ to after intervention $(M=86.8)$ by 2.6 points. The Wilcoxon Signed-Rank Test analysis showed this increase was not statistically significant $(Z=-1.35, P=.89)$.

Table 5

Comparison of Pre-test and Post-test PWBS Scores

\begin{tabular}{|c|c|c|}
\hline \multirow{2}{*}{ Participant } & \multicolumn{2}{|c|}{ Psychological Well-being } \\
\hline & Pre-test & Post-test \\
\hline A & 89 & 93 \\
\hline B & 80 & 81 \\
\hline C & 80 & 82 \\
\hline $\mathrm{D}$ & 82 & 77 \\
\hline $\mathrm{E}$ & 90 & 101 \\
\hline Mean & 84.2 & 86.8 \\
\hline Z & & \\
\hline Sig. $(p<.05)$ & & \\
\hline
\end{tabular}


Individual Results

Participant $A$

During the intervention process, participant $A$ was cooperative and open to share his opinions or respond to other participants. Qualitatively, participant A felt that the intervention helped him to identify his problems, and better understand his thoughts and feelings he had about his problems. This in turn helped him to evaluate and solve his problems more objectively.

\section{Participant $B$}

During the intervention process, participant B was more secluded and silent than other participants. Participant B admitted that initially he was hesitant to share his problems, worried that it will be reported to his supervisor.As the intervention progressed, participant B seemed to be more relaxed and open to share his problems and opinions. Participant $B$ reported that through the intervention process, he was able to reflect on himself, especially his thoughts and feelings, when experiencing stress. Participant B who felt he is easily irritable and angry reported feeling more confident that he could apply the mindfulness techniques to help him calm down during stress or under pressure.

\section{Participant $C$}

Participant $C$ was cooperative and opens throughout the intervention, and encouraged other participants to share their problems and opinions in the group. Qualitatively, participant $\mathrm{C}$ reported feeling more confident to regulate his stress, and the meditation techniques he learned could help him prevent feeling overwhelmed at work. Participant C considered the "mindful breathing" meditation as the easiest and most beneficial to apply when experiencing negative emotions.

\section{Participant D}

Participant $D$ was the only female participant in this study. Most of the time during the intervention process, participant $D$ showed enthusiasm and was open to share her opinions, and was often seen to take notes during the explanation from the therapist. 
Participant D showed improvement for her burnout, however, her psychological wellbeing assessment showed a decrease. This may be explained by the observation in the last session, participant $D$ seemed to be upset and quieter than in previous sessions. Also, participant $E$ informed the therapist that participant $D$ was going through a personal problem that she did not want to share with the group. Qualitatively, participant D reported gaining insight to appreciate her more, and to be more loving and forgiving of herself and others. Participant $D$ also felt that the intervention helped her to identify her thoughts and feelings when experiencing stress or negative emotions.

\section{Participant $E$}

Participant E was cooperative during intervention, and showed initiative to ask questions throughout the intervention. Participant E scored the lowest burnout level among other participants in the pre-test assessment. Qualitatively, participant $E$ felt that the intervention helped him to manage his negative thoughts and emotions, especially those related to uncontrollable events happening in his life. Participant $E$ also thought that the techniques taught in the intervention were simple enough to be applied daily on his own, so that he felt confident that he would be able to practice mindfulness and regulate his stress in the future.

In general, all participants understood the concept of mindfulness introduced in this intervention. Although in the first session not all participants quite understood the concept, through a group discussion (led by the therapist) they were all able to understand in the simple way of being aware and attentive to their thoughts, feelings, and physical sensations as they happened. At the last session, in the closing discussion, all participants considered the meditation techniques introduced and exercised in the intervention were quite easy to understand and practiced daily, such as the "mindful check-in”, "mindful breathing”, and "is your body happy?" meditations. The difficulty they reported when practicing the meditations is that they are easily distracted or their mind wandered. However, it was also explained to them that when they were aware of their mind-wandering, they were still practicing their mindfulness skill nevertheless. 


\section{Follow-Up Session}

Two weeks after the intervention ended, a follow-up session was conducted and measured the burnout and psychological well-being levels again. The results obtained from the follow-up assessments were compared with the post-test results at the end of the intervention. Statistical analysis using Wilcoxon Signed-Rank Test showed this decrease was not significant $(Z=-1.46 \mathrm{I}, \mathrm{P}=.144)$. Scores in the depersonalization domain showed a small increase from the post-test $(M=3.6)$ to the follow-up $(M=3.8)$. This increase was not statistically significant $(Z=-1.00, p=.317)$. In the personal accomplishment domain, there was an increase of score from the post-test $(M=40.4)$ to the follow-up $(M=4 I)$. This increase was also not statistically significant $(Z=.00, p=1.00)$.

For the psychological well-being assessment, results showed that the average score from the post-test $(M=86.8)$ decreased in the follow-up measurement $(M=83.4)$ however, this decrease was not statistically significant $(Z=-1.46 I, p=.144)$. This result indicated that even when the intervention seemed to have a small effect in increasing the psychological well-being of the participants, the effect did not last up to two weeks after intervention ended.

\section{Discussion}

This study found that group intervention using Mindfulness-Based Stress can reduce the level of burnout (decreasing scores of emotional exhaustion and depersonalization, and increasing score of personal accomplishment) for the participants in this study. However, through statistical analysis, it was found that this decrease was not significant, and only the depersonalization and personal accomplishment domains were found approaching significance.

This result may be caused by the small sample size in the study. This was compared to previous researches using MBSR for healthcare workers (Cohen-Katz et al., 2005; Shapiro et al., 2005; Mackenzie, et al., 2006) which uses larger sample size $(n>20)$, showed 
significant effects in reducing burnout. Furthermore, systematic review on burnout interventions for nursing home staff (Westermann, Kozak, Harling, \& Nienhaus, 20I4) found that the lack of effect found in some interventions is caused by a short period of the programs. Short-term measurements are difficult to prove a longer lasting effect for reducing stress and burnout than long-term measurements. A refresher course given regularly is also considered important to maintain the positive effects of interventions (Westermann et al., 2014). In this study, the lack of time to give a longer-duration program could also explain the insignificant result for reducing burnout in participants.

However, a trend of positive improvement was observed in this study, as shown by overall decreased mean of burnout. This trend may be further improved if the MBSR intervention was conducted longer (for example, conducting a typical 8 week program), giving the participants more time to practice. It may also be beneficial to obtain more participants and conduct a randomized controlled study design, to establish the efficacy of MBSR for reducing burnout.

Furthermore, this study also examined the application of MBSR in enhancing the participants' psychological well-being. Results showed that there are increased scores in general across participants in the pre-test and post-test measurements. However, this increase did not last up to two weeks in the follow-up measurement, where a decrease was found in the mean scores. Based on theoretical reviews, many factors contribute to the psychological well-being of an individual contributing to an individual's psychological well-being. The non-lasting effect of MBSR in this study is likely influenced by a variety of other factors outside the intervention, such as interpersonal relationship or financial conditions (Hidalgo et al., 20l0), or other personal matters outside of work.lt can be concluded that although MBSR group intervention are effective in reducing burnout with lasting effect among professional caregivers, the same effect was not found in increasing psychological well-being. 
There are several limitations in this study. First, the participants in this study were obtained through recommendation from the institution, Budi Mulia 3, based on work performance evaluation. This limits the opportunity for the researcher to do an initial screening procedure for the burnout and psychological well-being level beforehand. This allows the possibility that there were other caregivers with higher burnout levels who were undetected and not selected to participate in the intervention. Second, the participants of this study were mostly male, and therefore making it unable to compare the gender or generalize the result based on one female participant in this study. Third limitation is regarding the limited time allocated by Budi Mulia 3. This caused less time for the participants to practice independently in between sessions. The time allocation also limited the duration of each session, and therefore required flexibility in adjusting the activities on each session. For future studies, researchers can coordinate better with the institution to communicate the needs of the study. Researchers can also conduct an initial screening before recruiting participants to further enrich the data obtained from the observations and interviews.

\section{Conclusion}

It can be concluded that group intervention using MBSR was not statistically significant to reduce the participants' burnout levels. However, there was a trend of positive improvement observed in each individual's scores. This result suggests that MBSR intervention is potentially beneficial to aid burnout in professional caregivers, especially in reducing feelings of depersonalization and increasing feelings of personal accomplishment.

\section{Acknowledgment}

Appreciation is given to Panti Sosial Tresna Werdha Budi Mulia 3 for their help and cooperation in the intervention process. 


\section{References}

Ahola, K., Toppinen-Tanner, S., \& Seppänen, J. (2017). Interventions to alleviate burnout symptoms and to support return to work among employees with burnout: Systematic review and meta-analysis. Burnout Research, 4, I-II.

Barber, C. E., \& Iwai, M. (1996). Role conflict and role ambiguity as predictors of burnout among staff caring for elderly dementia patients. Journal of Gerontological Social Work, 26(I-2), I0I-I I6.

Brown, K. W., Ryan, R. M., \& Creswell, J. D. (2007). Mindfulness: Theoretical foundations and evidence for its salutary effects. Psychological Inquiry, I8(4), 2 I I-237.

Cocco, E. (2010). How much is geriatric caregivers burnout caring-specific? Questions from a questionnaire survey. Clinical practice and epidemiology in mental health: CP \& $E M H, 6,66$.

Cohen-Katz, J., Wiley, S. D., Capuano, T., Baker, D. M., \& Shapiro, S. (2005). The effects of mindfulness-based stress reduction on nurse stress and burnout, Part II: A quantitative and qualitative study. Holistic Nursing Practice, 19(I), 26-35.

Gosseries, O., Demertzi, A., Ledoux, D., Bruno, M. A., Vanhaudenhuyse, A., Thibaut, A., Laureys, S. \& Schnakers, C. (20I2). Burnout in healthcare workers managing chronic patients with disorders of consciousness. Brain Injury, 26(I2), I493-I 499.

Hidalgo, J. T., Bravo, B. N., Martínez, I. P., Pretel, F. A., Postigo, J. M., \& Rabadán, F. E. (2010). Psychological well-being, assessment tools and related factors. IE Wells, Psychological Well Being, 77-II3.

Jakarta Open Data. (20I8). Data jumlah warga binaan panti sosial bulan Desember 2018. Retrieved from http://data.jakarta.go.id/dataset/data-jumlah-wbs-pantisosial/resource/02e092b4-e42 I-4b0 I-aa99-4e l c000da le2

Kabat-Zinn, J. (2003). Mindfulness-based stress reduction (MBSR). Constructivism in the Human Sciences, 8(2), 73-82.

Lizano, E. L. (2015). Examining the impact of job burnout on the health and well-being of human service workers: A systematic review and synthesis. Human Service Organizations: Management, Leadership \& Governance, 39(3), I67-I8I. 
Mackenzie, C. S., Poulin, P. A., \& Seidman-Carlson, R. (2006). A brief mindfulness-based stress reduction intervention for nurses and nurse aides. Applied Nursing Research, 19(2), 105-109.

Marsaoly, Y. A. (200I). Peranan pekerja social dalam pelayanan social untuk memenuhi kebutuhan lanjut usia: Studi di Panti Sosial Tresna Werdha "Budi Mulia" Jakarta Timur (Doctoral dissertation, FISIP-UI, 200I).

Maslach, C., Jackson, S. E., \& Leiter, M. P. (1996). The Maslach burnout inventory manual.

Maslach, C., Schaufeli, W. B., \& Leiter, M. P. (200I). Job burnout. Annual Review Of Psychology, 52(I), 397-422.

Maslach, C., \& Leiter, M. P. (2016). Burnout. In Stress: Concepts, cognition, emotion, and behavior (pp. 35 I-357). Academic Press.

Nowrouzi, B., Lightfoot, N., Larivière, M., Carter, L., Rukholm, E., Schinke, R., \& BelangerGardner, D. (20I5). Occupational stress management and burnout interventions in nursing and their implications for healthy work environments: A literature review. Workplace Health \& Safety, 63(7), 308-3I5.

Nyklíček, I., \& Kuijpers, K. F. (2008). Effects of mindfulness-based stress reduction intervention on psychological well-being and quality of life: is increased mindfulness indeed the mechanism? Annals of Behavioral Medicine, 35(3), 33 I-340.

Putri, U. N. (2017). Mindfulness-based stress reduction (MBSR) untuk menurunkan tingkat stress pada care giver informal skizofrenia. (Thesis, Universitas Indonesia, 2017).

Ryff, C. D., \& Keyes, C. L. M. (1995). The structure of psychological well-being revisited. Journal of Personality and Social Psychology, 69(4), 719.

Shapiro, S. L., Astin, J. A., Bishop, S. R., \& Cordova, M. (2005). Mindfulness-based stress reduction for health care professionals: results from a randomized trial. International Journal of Stress Management, I2(2), I64- 178. 
Shapiro, S. L., Brown, K. W., \& Biegel, G. M. (2007). Teaching self-care to caregivers: Effects of mindfulness-based stress reduction on the mental health of therapists in training. Training and Education in Professional Psychology, I(2), I05-I I5. 